Paediatr Paedolog 2015 · 50:185 DOI 10.1007/s00608-015-0316-x Online publiziert: 15 . Oktober 2015 (c) Springer-Verlag Wien 2015

CrossMark

\title{
Wolfgang Sperl
}

Präsident der Österreichischen Gesellschaft für Kinder- und Jugendheilkunde, Univ.-Klinik für Kinder- und Jugendheilkunde, SALK, Salzburg, Österreich

\section{Kinder und Jugendliche auf der Flucht}

Richtlinien wurden erarbeitet, an wichtige Stellen verteilt und sollen demnächst auch in „pädiatrie \& pädologie “veröffentlicht werden.

grantenkinder ist eine große aktuelle Herausforderung.

In der Betreuung der Kinder und Jugendlichen leisten viele Kolleginnen und Kollegen im niedergelassenen und Krankenhausbereich großartige Arbeit. Ich möchte mich an dieser Stelle bei allen, die sich in der Versorgung der Flüchtlingskinder, u. a. in Wien, Nickelsdorf, Salzburg freiwillig, unentgeltlich, teilweise selbstorganisiert mit viel Empathie vorbildlich eingesetzt haben, herzlich bedanken!

Gerade in dieser Situation wurde und wird deutlich bewiesen, wie wichtig die Kinder- und Jugendfachärzte neben den Allgemeinmedizinern in der Grundversorgung sind, gerade in der Betreuung der Altersgruppe 0-18 Jahre.

\section{Tatkräftige Hilfe}

Die staatliche beauftragte Schweizer Organisation ORS wäre ohne die tatkräftige Hilfe der Kinder- und Jugendfachärztinnen nicht ausgekommen, wir haben den offiziellen Einsatz unser Kollegen und Kolleginnen mehrfach gefordert.

Doz. Dr. Nicole Grois hat sich über die Politische Kindermedizin (AG Medizinische Versorgung Flüchtlingskinder) und im Rahmen der ÖGKJ (Referat Transkulturelle Pädiatrie) sehr eingebracht und wertvolle Arbeit geleistet. Medizinische

\section{Zwei Kernprobleme}

Es sind zwei aktuelle Probleme, die anstehen: einerseits die Gefahr - bei Akkumulation von Asylwerbern und deren Familien und Kindern - gerade jetzt bei Beginn der kalten Jahreszeit, den Ausbruch von (Infektions-)Krankheiten zu kontrollieren und adäquat damit umzugehen. Hier sind vorausschauend medizinische Richtlinien notwendig und kompetentes Handeln angezeigt. Andererseits sind Kinder und Jugendliche von Asylwerbern oder auch zahlreiche alleinstehende Jugendliche als Mitbewohner „medizinisch“ in unser Land zu integrieren, und es ist eine adäquate Versorgung anzubieten.

\section{Untersuchungen und Dokumentation}

Es braucht definierte bundesweit abgestimmte Einstiegsuntersuchungen für alle Altersgruppen, standardisierte Eintrittsuntersuchungen in Kindergarten oder Schule. Wichtig ist eine von Beginn ebenfalls einheitliche Dokumentation der medizinischen Befunde und Handlungen, auf die alle in der Folge Bezug nehmen können. Wichtige Themen, wie die Nachholimpfungen bis hin zu selek- tiven Screening-Untersuchungen, sind zu klären, insbesondere deren Finanzierung.

Die Wertschätzung und Annahme von Asylwerbern und deren Kindern und Jugendlichen muss sich bei der medizinischen Versorgung ausdrücken.

Mit kollegialen Grüßen

Wolfgang Sperl

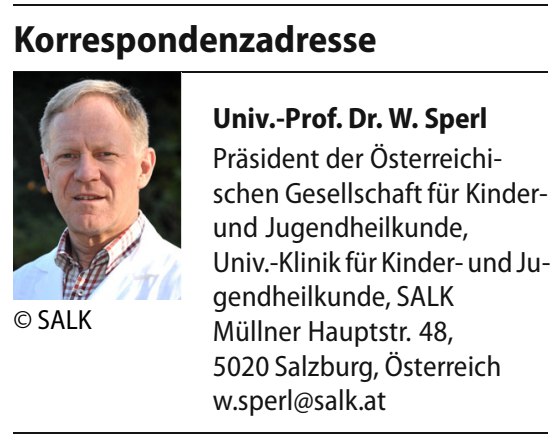

Interessenkonflikt. W. Sperl gibt an, dass kein Interessenkonflikt besteht. 